09.7

\title{
Энергопотребление при высокочастотной модуляции неохлаждаемого InGaAs/GaAs/AIGaAs-микродискового лазера
}

\author{
(C) А.Е. Жуков ${ }^{1}$, Э.И. Моисеев ${ }^{1}$, А.М. Надточий ${ }^{1}$, А.С. Драгунова ${ }^{1}$, Н.В. Крыжановская ${ }^{1}$, \\ М.М. Кулагина ${ }^{2}$, С.А. Минтаиров ${ }^{2}$, Н.А. Калюжный ${ }^{2}$, Ф.И. Зубов ${ }^{3}$, М.В. Максимов ${ }^{3}$ \\ ${ }^{1}$ Национальный исследовательский университет „Высшая школа экономики“, Санкт-Петербург, Россия \\ ${ }^{2}$ Физико-технический институт им. А.Ф. Иофффе РАН, Санкт-Петербург, Россия \\ ${ }^{3}$ Санкт-Петербургский национальный исследовательский Академический университет им. Ж.И. Алфёрова РАН, \\ Санкт-Петербург, Россия \\ E-mail: zhukale@gmail.com
}

Поступило в Редакцию 12 января 2021 г.

В окончательной редакции 2 апреля 2021 г.

Принято к публикации 2 апреля 2021 г.

Исследовано потребление энергии микродисковым лазером с квантовыми ямами-точками InGaAs/GaAs при высокочастотной модуляции без принудительного охлаждения. Для микролазера диаметром $20 \mu \mathrm{m}$ оценено наименьшее энергопотребление $1.6 \mathrm{pJ}$ в расчете на один бит данных, переданных с помощью оптического сигнала.

Ключевые слова: высокочастотная модуляция, микролазер, квантовые точки, энергопотребление.

DOI: 10.21883/PJTF.2021.13.51118.18707

Микродисковые / микрокольцевые лазеры [1,2] (МДЛ/МКЛ), в которых высокая добротность лазерной моды достигается за счет полного внутреннего отражения света от боковых стенок микрорезонатора, обладают по сравнению с вертикально-излучающими лазерами рядом преимуществ: для их создания может использоваться лазерная гетероструктура без распределенных брэгговских отражателей, подобная применяемой в полосковых лазерах; вывод излучения реализуется в плоскости подложки, и, следовательно, для интеграции с другими оптоэлектронными элементами не требуется применения дополнительных сложных в изготовлении элементов, таких как $45^{\circ}$ микрозеркала [3], интегральные дифракционные решетки [4], микропризмы [5]; процесс изготовления отличается простотой, поскольку включает одну операцию травления и не требует селективного окисления, пассивации или покрытия защитным диэлектриком. Были продемонстрированы МДЛ/МКЛ с квантовыми точками, монолитно [6,7] и гибридно [8] интегрированные с кремниевой подложкой. Высокочастотные характеристики таких микролазеров, хотя и уступают характеристикам вертикально-излучающих, позволяют осуществить безошибочную передачу данных со скоростью по крайней мере $10 \mathrm{Gbit} / \mathrm{s}$ [9].

Мерой энергоэффективности лазера, используемого для оптической передачи данных, является электрическая энергия, расходуемая в расчете на один бит переданной информации (energy-to-data ratio, EDR) [10]. Для микроспиральных температурно-стабилизированных лазеров на основе AlGaInAs/InP с радиусом 30-40 $\mu \mathrm{m}$ сообщалось о EDR в диапазоне 6.9-27.4 pJ/bit [11]. На основании данных, приведенных для неохлаждаемого микрокольцевого лазера с квантовыми точками
InAs/GaAs диаметром $30 \mu \mathrm{m}$ [12], можно оценить его энергопотребление как 3.4-13.9 pJ/bit в зависимости от тока. Недавно нами было продемонстрировано снижение потребления электроэнергии до $1.6 \mathrm{pJ} / \mathrm{bit}$ для микролазера диаметром $23 \mu \mathrm{m}$ при стабилизированной температуре $18^{\circ} \mathrm{C}$ [13]. Однако использование массивного теплоотвода и термоэлектрического охладителя не только ведет к дополнительному расходу энергии, но и в значительной степени затрудняет интеграцию микролазера с другими функциональными элементами. Для микролазеров диаметром 10.5, 19 и $23 \mu \mathrm{m}$, работающих без принудительного охлаждения, было оценено наименьшее энергопотребление $1.6,2.3$ и 2.8 pJ/bit соответственно при наибольшей частоте модуляции 3.8, 5 и $5.2 \mathrm{GHz}[14]$.

Снижение EDR и увеличение скорости модуляции требует, в частности, как снижения порогового тока, так и подавления роста тока спонтанной рекомбинации в запороговом режиме, вызванного саморазогревом микролазера [15]. В настоящей работе нами демонстрируется снижение теплового сопротивления микродисковых лазеров, позволившее достичь EDR $1.6 \mathrm{pJ} / \mathrm{bit}$ и наибольшей ширины полосы модуляции $5.9 \mathrm{GHz}$ в микродисковом лазере диаметром $20 \mu \mathrm{m}$, работающем без температурной стабилизации, т.е. без какого-либо принудительного охлаждения.

Лазерная гетероструктура была выращена методом газофазной эпитаксии из металлоорганических соединений на слаборазориентированной относительно (100) подложке $n^{+}$-GaAs. Пять слоев квантовых ям-точек $\mathrm{In}_{0.4} \mathrm{Ga}_{0.6} \mathrm{As} / \mathrm{GaAs}$ [16] помещены в волновод $\mathrm{GaAs}$ толщиной около $0.7 \mu \mathrm{m}$, ограниченный эмиттерами $\mathrm{Al}_{0.39} \mathrm{Ga}_{0.61}$ As. Микродисковые резонаторы были изго- 
товлены с помощью фотолитографии и плазмохимического травления. Индивидуальные контакты $p$-типа были сформированы на вершинах мез, после чего микродиски были планаризованы с помощью эпоксидного фоторе-

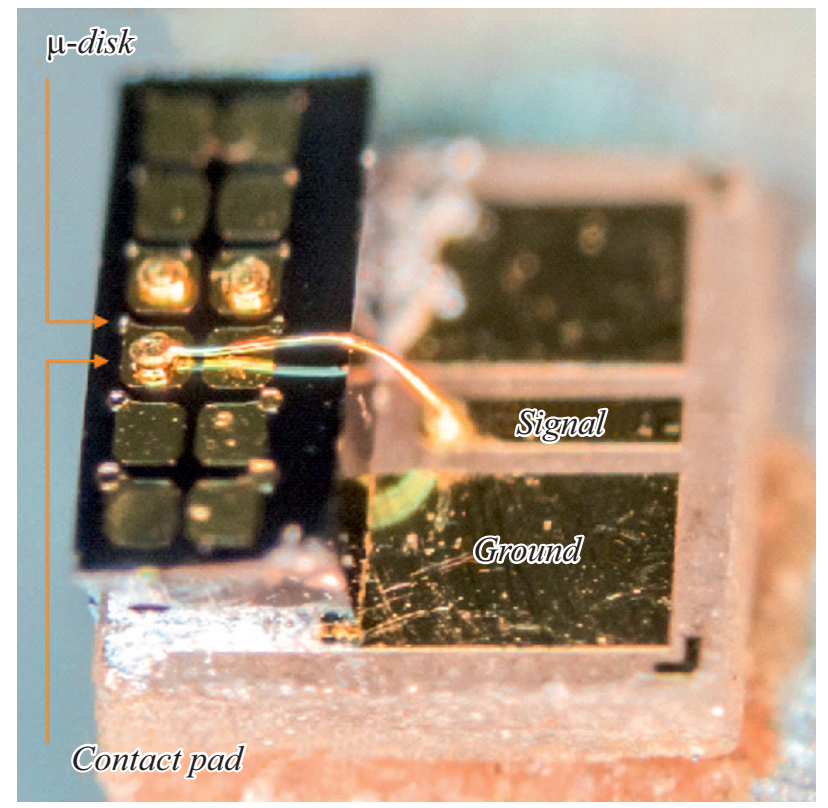

Рис. 1. Чип с массивом микролазеров, смонтированный на СВЧ-плату. зиста SU-8, поверх которого были изготовлены вспомогательные контактные площадки $(\sim 120 \times 120 \mu \mathrm{m})$. Чипы с микролазерами монтировались на СВЧ-платы $n$-контактом, сформированным на задней стороне утоненной подложки. С использованием установки микросварки ТРТ НВ16 к вспомогательным контактным площадкам прикреплялись золотые проволоки диаметром $17 \mu \mathrm{m}$ и длиной около $500 \mu \mathrm{m}$ от сигнального контакта СВЧ-плат (рис. 1).

Исследовался микродисковый лазер диаметром $20 \mu \mathrm{m}$, измерения проводились при комнатной температуре без термостабилизации и какого-либо принудительного охлаждения микролазера. Вольт- и ватт-амперные характеристики измерялись в непрерывном режиме. Выходная мощность, а также спектры излучения регистрировались с помощью анализатора оптических спектров Yokogawa AQ6370C. Малосигнальная прямая модуляция была реализована с использованием зонда типа ground-signal-ground. Частота варьировалась в пределах $0.5-10 \mathrm{GHz}$, ток смещения - от 4 до $15 \mathrm{~mA}$. Измерения проводились с помощью фотодетектора New Focus 1434 с полосой пропускания $25 \mathrm{GHz}$ и анализатора цепей Agilent E8364B.

Пороговый ток, равный $4 \mathrm{~mA}$, был определен по излому ватт-амперной характеристики (рис. 2). При этом токе в спектре излучения начинает отчетливо проявляться лазерная линия с длиной волны около $1096 \mathrm{~nm}$

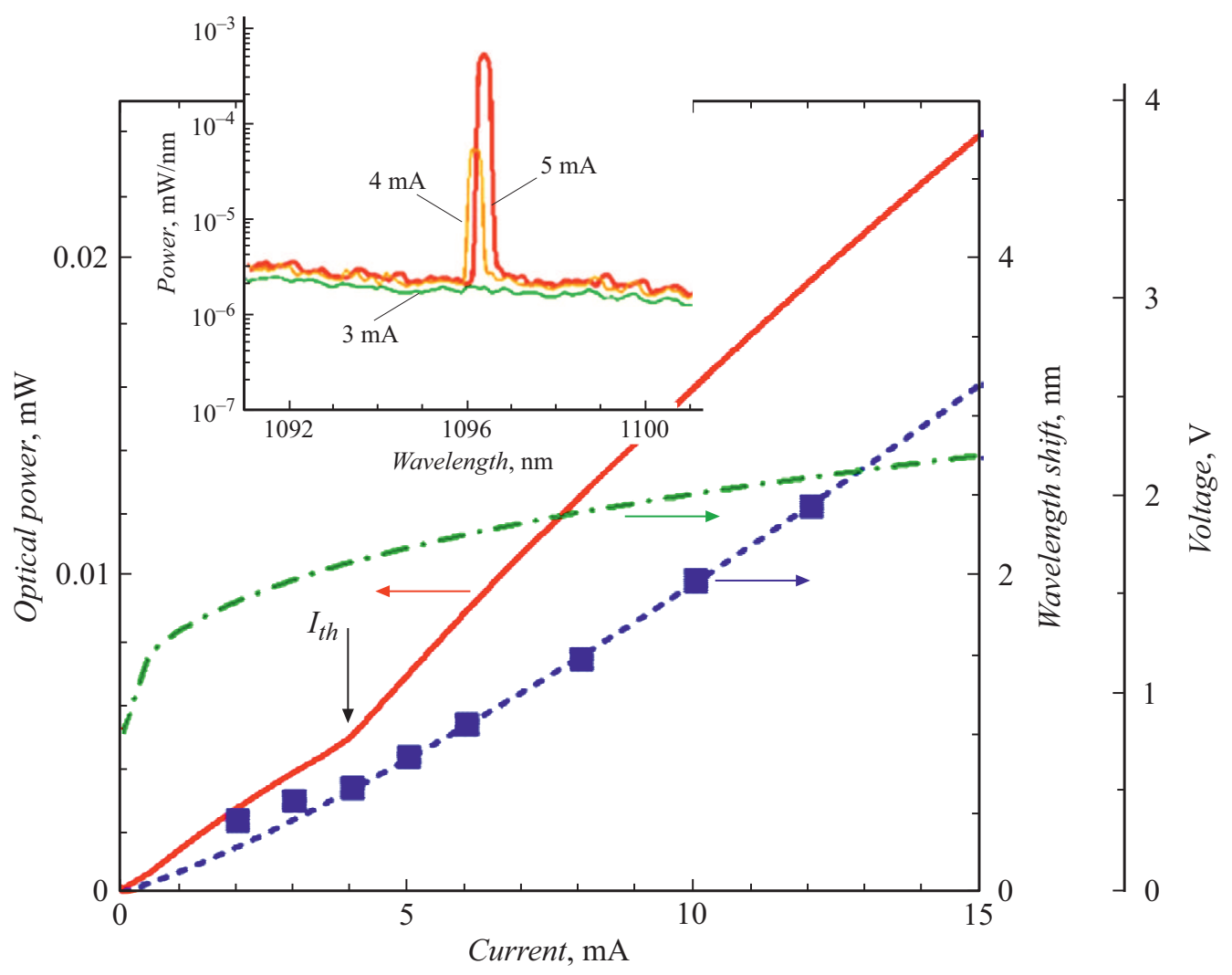

Рис. 2. Оптическая мощность (сплошная линия), напряжение (штрихпунктирная линия) и сдвиг линии генерации (символы эксперимент, штриховая линия- аппроксимация) в зависимости от тока. На вставке - спектры излучения при различных токах вблизи порога генерации. 


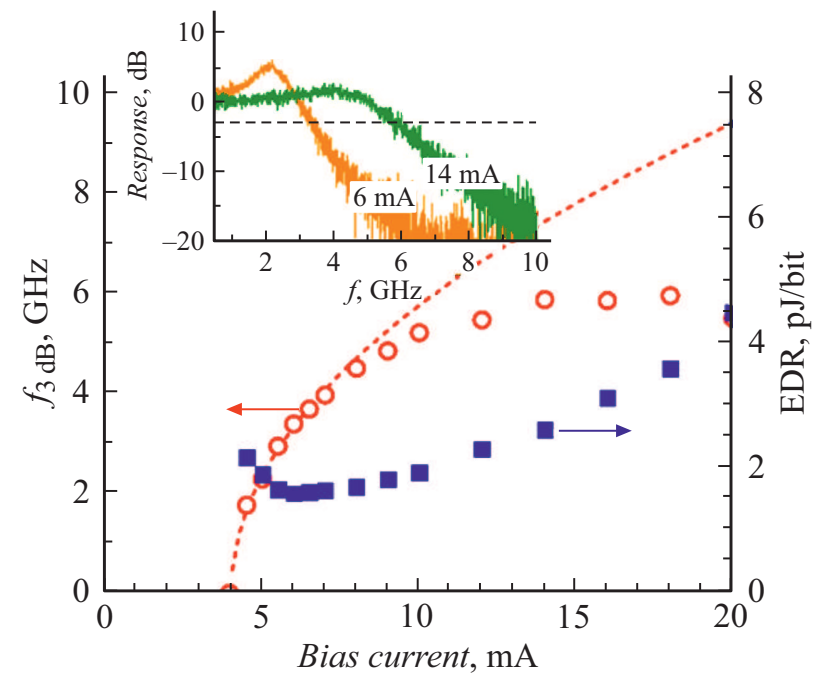

Рис. 3. Зависимость полосы модуляции по уровню $-3 \mathrm{~dB}$ (кружки - эксперимент, линия - аппроксимация вблизи порогового тока зависимостью $\left.m \sqrt{I-I_{t h}}\right)$ и величины электрических потерь при переключении (квадраты) от тока смещения. На вставке - амплитудно-частотная характеристика при различных токах смещения.

(полная ширина на половине высоты $0.25 \mathrm{~nm}$ ограничена спектральным разрешением) (вставка к рис. 2). При увеличении тока инжекции $I$ максимум линии генерации сдвигается в длинноволновую сторону. Сдвиг линии пропорционален потребляемой электрической мощности $U I$ (здесь $U-$ напряжение, определяемое вольт-амперной характеристикой микролазера), коэффициент пропорциональности определен равным $0.097 \mathrm{~nm} / \mathrm{mW}$. С учетом величины температурного коэффициента моды шепчущей галереи около $0.08 \mathrm{~nm} / \mathrm{K}$ [17] было вычислено тепловое сопротивление отводу тепла от активной области $1.21^{\circ} \mathrm{C} / \mathrm{mW}$. Это соответствует величине удельного теплового сопротивления $3.8 \cdot 10^{-3 \circ} \mathrm{C} \cdot \mathrm{cm}^{2} / \mathrm{W}$, тогда как для ранее исследованных микролазеров с квантовыми точками эта величина составила $5 \cdot 10^{-3 \circ} \mathrm{C} \cdot \mathrm{cm}^{2} / \mathrm{W}[18]$. Улучшение теплового сопротивления мы связываем с дополнительным отводом тепла посредством верхних контактных площадок.

При некотором токе смещения полоса модуляции $f_{3 \mathrm{~dB}}$ микролазера определялась по спаду модуляционного отклика в 2 раза (до уровня $-3 \mathrm{~dB}$ ) относительно его низкочастотного значения (вставка к рис. 3). Вблизи порога генерации выполняется соотношение $f_{3 \mathrm{~dB}} \approx m \sqrt{I-I_{t h}}$, где описывающий эффективность токовой модуляции коэффициент $m$ был определен равным $2.4 \mathrm{GHz} / \mathrm{mA}^{0.5}$. Наибольшая ширина полосы модуляции составила $5.9 \mathrm{GHz}$ (рис. 3).

Энергия $\mathrm{EDR}=U I / B$, потребляемая лазером для передачи одного бита информации, по определению есть отношение электрической мощности к скорости передачи битов $B$ [19], которая была оценена по удвоенной полосе модуляции $B=2 f_{3 \mathrm{~dB}}$ [20]. Наименьшее значение
EDR, достигаемое при токе смещения $6 \mathrm{~mA}$, равно $1.6 \mathrm{pJ} / \mathrm{bit}$. При этом токе величина перегрева микролазера относительно окружающей среды, оцененная с учетом теплового сопротивления, составляет около $13^{\circ} \mathrm{C}$. При уменьшении тока смещения относительно его оптимального значения величина EDR растет вследствие быстрого спада полосы модуляции при приближении к пороговому току. При увеличении тока EDR возрастает по причине более быстрого увеличения $U I$ по сравнению с $f_{3 \mathrm{~dB}}$, в том числе вследствие увеличивающегося перегрева микролазера, ведущего к насыщению полосы модуляции.

Таким образом, нами были исследованы электрические потери микродискового лазера $\mathrm{AlGaAs} / \mathrm{GaAs}$ c квантовыми ямами-точками InGaAs, работающего без принудительного охлаждения. Снижение удельного теплового сопротивления позволило увеличить максимальную частоту модуляции при одновременном снижении энергопотребления по сравнению с ранее продемонстрированными неохлаждаемыми микролазерами. Достигнуто потребление энергии в расчете на один бит, сравнимое со значениями, ранее продемонстрированными для температурно-стабилизированных микродисковых лазеров.

\section{Финансирование работы}

Работа выполнена при поддержке Российского научного фонда (соглашение № 19-72-30010). Оптические исследования проведены в рамках Программы фундаментальных исследований НИУ ВШЭ.

\section{Конфликт интересов}

Авторы заявляют, что у них нет конфликта интересов.

\section{Список литературы}

[1] S.L. McCall, A.F.J. Levi, R.E. Slusher, S.J. Pearton, R.A. Logan, Appl. Phys. Lett., 60 (3), 289 (1992). DOI: $10.1063 / 1.106688$

[2] M.-H. Mao, H.-C. Chien, J.-Z. Hong, C.-Y. Cheng, Opt. Express, 19 (15), 14145 (2011). DOI: 10.1364/OE.19.014145

[3] P.-K. Shen, C.-T. Chen, C.-H. Chang, C.-Y. Chiu, C.-C. Chang, H.-C. Lan, Y.-C. Lee, M.-L. Wu, in Optical Fiber Communication Conference 2014 (OFC 2014) (San Francisco, 2014), Paper M2K.6. DOI: 10.1364/OFC.2014.M2K.6

[4] D.A. Louderback, G.W. Pickrell, H.C. Lin, M.A. Fish, J.J. Hindi, P.S. Guilfoyle, Electron. Lett., 40 (17), 1064 (2004). DOI: 10.1049/el:20045585

[5] K.S. Kaur, A.Z. Subramanian, P. Cardile, R. Verplancke, J. van Kerrebrouck, S. Spiga, R. Meyer, J. Bauwelinck, R. Baets, G. van Steenberge, Opt. Express, 23 (22), 28264 (2015). DOI: 10.1364/OE.23.028264

[6] N. Kryzhanovskaya, E. Moiseev, Yu. Polubavkina, M. Maximov, M. Kulagina, S. Troshkov, Yu. Zadiranov, Yu. Guseva, A. Lipovskii, M. Tang, M. Liao, J. Wu, S. Chen, H. Liu, A. Zhukov, Opt. Lett., 42 (17), 3319 (2017). DOI: $10.1364 /$ OL.42.003319 
[7] Y. Wan, J. Norman, Q. Li, M.J. Kennedy, D. Liang, C. Zhang, D. Huang, Z. Zhang, A.Y. Liu, A. Torres, D. Jung, A.C. Gossard, E.L. Hu, K.M. Lau, J.E. Bowers, Optica, 4 (8), 940 (2018). https://doi.org/10.1364/OPTICA.4.000940

[8] A.E. Zhukov, N.V. Kryzhanovskaya, E.I. Moiseev, A.S. Dragunova, M. Tang, S. Chen, H. Liu, M.M. Kulagina, S.A. Kadinskaya, F.I. Zubov, A.M. Mozharov, M.V. Maximov, Materials, 13 (10), 2315 (2020). https://doi.org/10.3390/ma13102315

[9] F. Zubov, M. Maximov, N. Kryzhanovskaya, E. Moiseev, M. Muretova, A. Mozharov, N. Kaluzhnyy, S. Mintairov, M. Kulagina, N. Ledentsov, Jr., L. Chorchos, N. Ledentstsov, A. Zhukov, Opt. Lett., 44 (22), 5442 (2019). https://doi.org/10.1364/OL.44.005442

[10] D.A.B. Miller, Proc. of the IEEE, 97 (7), 1166 (2009). DOI: 10.1109/JPROC.2009.2014298

[11] Y.-D. Yang, Y. Zhang, Y.-Z. Huang, A.W. Poon, Opt. Express, 22 (1), 824 (2014). https://doi.org/10.1364/OE.22.000824

[12] Y. Wan, D. Inoue, D. Jung, J.C. Norman, C. Shang, A.C. Gossard, J.E. Bowers, Photon. Res., 6 (8), 776 (2018). https://doi.org/10.1364/PRJ.6.000776

[13] А.Е. Жуков, Э.И. Моисеев, Н.В. Крыжановская, Ф.И. Зубов, А.М. Можаров, Н.А. Калюжный, С.А. Минтаиров, М.М. Кулагина, С.А. Блохин, М.В. Максимов, Письма в ЖТФ, 45 (16), 49 (2019).

DOI: 10.21883/PJTF.2019.16.48158.17885 [Пер. версия: 10.1134/S1063785019080315].

[14] N.V. Kryzhanovskaya, E.I. Moiseev, F.I. Zubov, A.M. Mozharov, M.V. Maximov, N.A. Kalyuzhny, S.A. Mintairov, Yu.A. Guseva, M.M. Kulagina, S.A. Blokhin, Yu. Berdnikov, A.E. Zhukov, J. Appl. Phys., 126 (6), 063107 (2019). https://doi.org/10.1063/1.5108556

[15] A.E. Zhukov, N.V. Kryzhanovskaya, E.I. Moiseev, A.M. Nadtochiy, A.S. Dragunova, M.V. Maximov, F.I. Zubov, S.A. Kadinskaya, Yu. Berdnikov, M.M. Kulagina, S.A. Mintairov, N.A. Kalyuzhnyy, IEEE J. Quantum Electron., 56 (5), 2000908 (2020). DOI: 10.1109/JQE.2020.3009954

[16] M.V. Maximov, A.M. Nadtochiy, S.A. Mintairov, N.A. Kalyuzhnyy, N.V. Kryzhanovskaya, E.I. Moiseev, N.Yu. Gordeev, Yu.M. Shernyakov, A.S. Payusov, F.I. Zubov, V.N. Nevedomskiy, S.S. Rouvimov, A.E. Zhukov, Appl. Sci., 10 (3), 1038 (2020). DOI: 10.3390/app10031038

[17] N.V. Kryzhanovskaya, E.I. Moiseev, Yu.V. Kudashova, F.I. Zubov, A.A. Lipovskii, M.M. Kulagina, S.I. Troshkov, Yu.M. Zadiranov, D.A. Livshits, M.V. Maximov, A.E. Zhukov, Electron. Lett., 51 (17), 1354 (2015). DOI: $10.1049 / \mathrm{el} .2015 .2325$

[18] Н.В. Крыжановская, М.В. Максимов, С.А. Блохин, М.А. Бобров, М.М. Кулагина, С.И. Трошков, Ю.М. Задиранов, А.А. Липовский, Э.И. Моисеев, Ю.В. Кудашова, Д.А. Лившиц, В.М. Устинов, А.Е. Жуков, ФТП, 50 (3), 393 (2016). [Пер. версия: 10.1134/S1063782616030131].

[19] P. Moser, W. Hofmann, P. Wolf, J.A. Lott, G. Larisch, A. Payusov, N.N. Ledentsov, D. Bimberg, Appl. Phys. Lett., 98 (23), 231106 (2011). https://doi.org/10.1063/1.3597799

[20] E. Kapon, in Semiconductor lasers I. Fundamentals (Academic Press, 1999), ch. 3.4. 